\title{
Predictive electroweak gauge model with strong spontaneous-symmetry-breaking dynamics
}

\author{
Petr Beneš, ${ }^{a}$ Jiří Hošek ${ }^{a, b, *}$ and Adam Smetana ${ }^{a}$ \\ ${ }^{a}$ Institute of Experimental and Applied Physics, Czech Technical University in Prague, \\ Husova 240/5, 11000 Prague 1, Czech Republic \\ ${ }^{b}$ Department of Theoretical Physics, Nuclear Physics Institute, Czech Academy of Sciences, $25068 \check{R} e z ̌$ \\ (Prague), Czech Republic
}

E-mail: petr.benes@utef.cvut.cz, hosek@ujf.cas.cz, adam.smetanar@cvut.cz

Higgs sector of the Standard model (SM) is replaced by quantum flavor dynamics (QFD), the gauged flavor $S U(3)_{f}$ symmetry with scale $\Lambda$. For all SM chiral fermions in triplets the anomaly freedom demands addition of a triplet of $v_{R}$. Approximate QFD Schwinger-Dyson equation for the Euclidean infrared fermion self-energies $\Sigma_{f}\left(p^{2}\right)$ has spontaneous-chiral-symmetry-breaking solutions ideal for seesaw: (1) $\Sigma_{f}\left(p^{2}\right)=M_{f R}^{2} / p$ where three Majorana masses $M_{f R}$ of $v_{f R}$ are of order $\Lambda$. (2) $\Sigma_{f}\left(p^{2}\right)=m_{f}^{2} / p$ where three Dirac masses $m_{f}=m_{(0)} 1+m_{(3)} \lambda_{3}+m_{(8)} \lambda_{8}$ of SM fermions are exponentially suppressed w.r.t. $\Lambda$, and degenerate for all SM fermions in $f$. $M_{f R}$ break $S U(3)_{f}$ completely, and $m_{(3)}, m_{(8)}$ superimpose its tiny breaking to $U(1) \times U(1)$. All flavor gluons thus acquire self-consistently the masses $\sim \Lambda . m_{f}$ break $S U(2)_{L} \times U(1)_{Y}$ to $U(1)_{e m}$. Symmetry partners of the composite 'would-be' Nambu-Goldstone bosons are the genuine Higgs particles: (1) Three $v_{R}$-composed Higgses $\chi_{i}$ with masses $\sim \Lambda$. (2) Two new SM-fermion-composed Higgses $h_{3}, h_{8}$ with masses $\sim m_{(3)}, m_{(8)}$, respectively. (3) The SM-like SM-fermion-composed Higgs $h$ with mass $\sim m_{(0)}$, the effective Fermi scale. Electroweak loops with $\Sigma_{f}\left(p^{2}\right)$-dependent vertices enforced by the symmetry of the Lagrangian generate the $W$ and $Z$ masses at Fermi scale, and provide the fermion mass splitting in $f$. At the present exploratory stage the splitting is unrealistic.

40th International Conference on High Energy physics - ICHEP2020

July 28 - August 6, 2020

Prague, Czech Republic (virtual meeting)

\footnotetext{
${ }^{*}$ Speaker
} 


\section{Introduction}

After the discovery of the Higgs boson at the LHC in 2012 the Higgs mechanism of the Standard model (SM) is fixed. As the mechanism of generation of all particle masses it is, however, certainly incomplete: (i) The neutrinos are massive.(ii) there is the dark matter. (iii) In a good quantum description of the world the fermion masses should be calculable [1]. Moreover, as pointed already by its authors the Higgs mechanism is perhaps also phenomenological. Then the Higgs boson is a fermion-antifermion composite and the weakly coupled SM Higgs sector is replaced by a new strong force. Although strong coupling dynamics is difficult to handle, we take this route [2].

We accept the argumentation of particularly economical class of models suggested long time ago by Heinz Pagels and his collaborators in [3], [4]: If a new strong dynamics (called by Pagels the 'quantum flavor dynamics', QFD) generates spontaneously the masses of leptons and quarks of the SM, then the electroweak $S U(2)_{L} \times U(1)_{Y}$ symmetry breaks spontaneously down to unbroken $U(1)_{e m}$. Hence, there are three composite Nambu-Goldstone (NG) bosons bound by the new strong force. The NG bosons are in fact 'would-be' ones, i.e., the $W$ and $Z$ bosons acquire masses. There is a massive composite Higgs boson as the genuine symmetry partner of the 'would-be' NG bosons. The fermion masses are calculable. Unlike the Higgs mechanism the role of the dynamical generation of the fermion masses is primary. Here we follow the particular suggestion of Tsutomu Yanagida [5]. We gauge the flavor $S U(3)_{f}$ symmetry. Therefore, the new dynamics strongly coupled in the infrared, is mediated by the octet of flavor gluons $C_{a}^{\mu}$. Putting all SM chiral fermion fields $f^{(\mathrm{SM})}$ in triplets we are enforced by the anomaly freedom to enlarge the fermion sector by one triplet of $v_{R}$. The resulting Lagrangian is

$$
\mathcal{L}=-\frac{1}{4} F_{a \mu \nu}^{A} F_{a}^{A \mu \nu}-\frac{1}{4} F_{\mu \nu}^{B} F^{B \mu \nu}-\frac{1}{4} F_{a \mu \nu}^{C} F_{a}^{C \mu \nu}+\bar{f}^{(\mathrm{SM})} i \not D f^{(\mathrm{SM})}+\bar{v}_{R} i \not D v_{R} .
$$

The $S U(3)_{f}$ interaction, characterized by one dimensionless coupling constant $h$, is asymptotically free at high momenta and hence strongly interacting in the infrared. This means that by dimensional transmutation the dimensionless $h$ can turn into the theoretically arbitrary mass scale $\Lambda$, the only free unknown parameter of the model. We find the Pagel's notion of 'quantum flavor dynamics' (QFD) very appropriate and take the liberty of using it to name also our gauge $S U(3)_{f}$ dynamics.

It is utmost important that the hard masses of all fermions are strictly prohibited by the symmetries of $\mathcal{L}$. The main value of the model is that without any fine tuning, the QFD SchwingerDyson equation, in a crude separable approximation, provides simultaneously three Majorana masses $M_{f R}$ of three $v_{f R}$, and three Dirac masses $m_{f}$, degenerate for all SM fermion species in $f$ with the property $M_{f R} \gg m_{f}$. We will argue that this will become more realistic if the fermion self-energies $\Sigma\left(p^{2}\right)$ are taken into account in the electroweak interactions. This is done by employing the new vertices in the electroweak Ward-Takahashi (WT) identities. First, the axialvector vertices are known to give rise to the $W, Z$ boson masses. Second, the polar-vector vertices are suggested for the fermion mass splitting within flavors.

\section{Spontaneous generation of Majorana and Dirac masses}

The universal QFD Schwinger-Dyson equation for the $\Sigma_{f}\left(p^{2}\right)$ of all full fermion propagators $S^{-1}(p)=\not p-\Sigma\left(p^{2}\right)$, can be formulated in the simplest approximation according to the same lines 
as in [6]

$$
\Sigma(p)=\int_{0}^{\Lambda} k^{3} d k K_{a b}(p, k) T_{a}(R) \Sigma(k)\left[k^{2}+\Sigma^{+} \Sigma\right]^{-1} T_{b}(L)
$$

where the unknown kernel is separately symmetric in momenta and in the flavor octet indices. Hence we make the BCS-motivated separable Ansatz

$$
K_{a b}(p, k)=\frac{3}{4 \pi^{2}} \frac{g_{a b}}{p k}
$$

Here $g_{a b}$ is a real symmetric matrix of the effective low-momentum dimensionless coupling constants. The integral equation is easily 'solved': $\Sigma \sim 1 / p$. The difficult part is to solve the non-linear algebraic equation for the matrix structure. Neglecting the flavor mixing (taking into account only $g_{11}=-g_{22}, g_{44}=-g_{55}, g_{66}=-g_{77}, g_{33}, g_{38}, g_{88}$ different from zero) we get [7]:

$$
M_{f R} \sim \Lambda, \quad m_{f}=\Lambda \exp \left(-1 / 4 \alpha_{\mathrm{ii}}\right) \text {, }
$$

where $\alpha_{11}=\frac{3}{64 \pi^{2}}\left(g_{33}+\frac{2}{\sqrt{3}} g_{38}+\frac{1}{3} g_{88}\right), \alpha_{22}=\frac{3}{64 \pi^{2}}\left(g_{33}-\frac{2}{\sqrt{3}} g_{38}+\frac{1}{3} g_{88}\right)$ and $\alpha_{33}=\frac{3}{64 \pi^{2}} \frac{4}{3} g_{88}$ are all positive and all $\alpha_{i i} \ll 1$.

The Majorana self-energies $\Sigma_{f}\left(p^{2}\right)=M_{f R}^{2} / p$ enter the effective Lagrangian as the diagonal entries of the complex sextet, composite of $v_{R}$. The Pauli principle selects from the decomposition $3^{*} \times 3^{*}=3+6^{*}$ only the symmetric representation. They break the symmetry $S U(3)_{f} \times U(1)$ of $\mathcal{L}$ completely. The consequence is two-fold: First, by the Goldstone theorem there are eight $v_{R}$-composed 'would-be' NG bosons and one pseudo-NG boson. This implies that all eight flavor gluons acquire masses of order $\Lambda$. The fate of the ninth NG boson of a global $U(1)$ symmetry is more subtle and is discussed in [7]. Second, according to NJL three remaining symmetry partners in the complex composite symmetric sextet $(2 \times 6=12=8+1+3)$ are interpreted as the genuine $v_{R}$-composed Higgs bosons with masses at scale $\Lambda$.

The Dirac self-energies $\Sigma_{f}\left(p^{2}\right)=m_{f}^{2} / p$ of all SM chiral fermion fields enter the effective Lagrangian as the diagonal entries of $3^{*} \times 3=1+8$ in the $S U(3)$ space. Obviously, each fermion mass $m_{f}$ breaks the electroweak symmetry $S U(2)_{L} \times U(1)_{Y}$ down to $U(1)_{e m}$ by a SM-fermion-composite multi-component doublet. Hence, by Goldstone theorem there are three composite multi-component 'would-be' NG bosons and, according to NJL, there is one composite multi-component genuine Higgs boson $h$ at the scale $\sim m_{(0)} \sim \sum_{f} m_{f}$. Since $m_{f}=m_{(0)} 1+m_{(3)} \lambda_{3}+m_{(8)} \lambda_{8}$, the fermion masses $m_{(3)}$ and $m_{(8)}$ break $S U(3)_{f}$ to $U(1) \times U(1)$ by a SM-fermion-composite multi-component octet. Hence, by Goldstone theorem there are six composite multi-component 'would-be' NG modes and, according to NJL, two composite multi-component genuine Higgs bosons $h_{3}$ and $h_{8}$ with masses $\sim m_{(3)}, m_{(8)}$, respectively.

\section{Consequences of the electroweak WT identities}

In general, the WT identities are the consequences of the symmetry of the Lagrangian. The $\Sigma_{f}\left(p^{2}\right)$ then inevitably induce new vertices in the WT identities in order to recover their validity, 
which (with $i=v, l, u, d$ ) have the form

$$
\begin{aligned}
\Gamma_{A}^{\mu}\left(p^{\prime}, p\right) & =e Q_{i}\left[\gamma^{\mu}-\left(p^{\prime}+p\right)^{\mu} \Sigma^{\prime}\left(p^{\prime}, p\right)\right] \\
\Gamma_{W}^{+\mu}\left(p^{\prime}, p\right) & =\frac{\sqrt{ } 2 e}{4 \sin \theta_{W}}\left\{\left[\gamma^{\mu}-\left(p^{\prime}+p\right)^{\mu} \Sigma^{\prime}\left(p^{\prime}, p\right)\right] T^{+}-\left[\gamma^{\mu} \gamma_{5} T^{+}-\frac{\left(p^{\prime}-p\right)^{\mu}}{\left(p^{\prime}-p\right)^{2}}\left(\Sigma\left(p^{\prime}\right)+\Sigma(p)\right) \gamma_{5} T^{+}\right]\right\} \\
\Gamma_{Z}^{\mu}\left(p^{\prime}, p\right) & =\frac{e}{\sin 2 \theta_{W}}\left\{\left[\gamma^{\mu}-\left(p^{\prime}+p\right)^{\mu} \Sigma^{\prime}\left(p^{\prime}, p\right)\right] T_{Z}^{i}-\left[\gamma^{\mu} \gamma_{5}-\frac{\left(p^{\prime}-p\right)^{\mu}}{\left(p^{\prime}-p\right)^{2}}\left(\Sigma\left(p^{\prime}\right)+\Sigma(p)\right) \gamma_{5}\right] T_{3 L}^{i}\right\},
\end{aligned}
$$

where the 'derivative' is defined as $\left.\Sigma^{\prime}\left(p^{\prime}, p\right) \equiv \Sigma\left(p^{\prime}\right)-\Sigma(p)\right) /\left(p^{\prime 2}-p^{2}\right)$ and $T_{Z}^{i} \equiv\left(T_{3 L}^{i}-\right.$ $\left.2 Q_{i} \sin ^{2} \theta_{W}\right)$. It in fact points to the difference between the $\Sigma\left(p^{2}\right)$-dependent terms in the axialvector and polar-vector vertices. In the former ones they mark the famous massless 'would-be' Nambu-Goldstone poles and persist even for constant $\Sigma$ 's i.e., for hard fermion masses. This is how the NG pole manifests in the NJL model. This robust effect is supported by the existence theorem. The polar-vector vertices appear only if the $\Sigma$ s are momentum-dependent. Consequently, their consequences depend crucially upon the functional form.

The $W$ and $Z$ boson masses are given by the Pagels-Stokar formula for the 'pion decay constant':

$$
F_{f}^{2}=8 N \int \frac{d^{4} p}{(2 \pi)^{4}} \frac{\Sigma_{f}^{2}\left(p^{2}\right)-\frac{1}{4} p^{2}\left(\Sigma_{f}^{2}\left(p^{2}\right)\right)^{\prime}}{\left(p^{2}+\Sigma_{f}^{2}\right)^{2}}
$$

Clearly, all fermion masses incoherently contribute. For $\Sigma_{f}\left(p^{2}\right)=m_{f}^{2} / p$ the integral is easily computed and the result is

$$
m_{W}^{2}=\frac{1}{4} g^{2} \frac{5}{4 \pi} \sum_{f} m_{f}^{2}, \quad m_{Z}^{2}=\frac{1}{4}\left(g^{2}+g^{\prime 2}\right) \frac{5}{4 \pi} \sum_{f} m_{f}^{2}
$$

The Weinberg relation $m_{W}=m_{Z} \cos \theta_{\mathrm{W}}$ is the consequence of the degeneracy of the fermion masses in electroweak doublets. It is due to the fact that all chiral fermion fields are in flavor triplets.

\section{SM fermion mass splitting in family}

Inspired by prescient Heinz Pagels who attempted to compute the $u-d$ quark mass difference due to the electromagnetic interaction [3] with new $\Sigma\left(p^{2}\right)$-dependent vertex we suggest here that the SM fermion mass splitting in families is entirely due to the electroweak interactions with peculiar vectorial $\Sigma\left(p^{2}\right)$-dependent vertices enforced by the WT identities [3], [8], [9].

The new polar-vector electroweak vertices are contained in $\Gamma_{A, Z, W}^{\mu}\left(p^{\prime}, p\right)$. Being chiralitychanging they are suited for contributing to fermion masses. An obvious objection is of course how it can be that the weakly coupled electroweak interaction describes the observed enormous top-bottom quark mass splitting. There is an argument: The computation of the fermion pole-mass splitting amounts to solving a complicated algebraic equation (10) which crucially depends upon the functional form of the resulting $\Sigma_{f}^{i}\left(p^{2}\right)$. It of course critically depends upon the functional form of $\Sigma_{f}\left(p^{2}\right)$. Another obvious objection is that the observed ordering of fermion masses in different families is different whereas the electroweak interactions are identical for all three families: In the first family the $u$ quark is lighter than the $d$ quark, whereas in other two families the corresponding ordering is dramatically reversed. In the second family the muon is heavier than the $s$ quark, whereas in other two families the corresponding ordering is reversed. There is an argument: The 
'known' parameters $m_{Z} / m_{f}$ and $m_{W} / m_{f}$ which come from QFD and from the axial-vector terms in the WT identities could account for these properties.

We take the massive propagators of $W$ and $Z$ bosons in the 'soft' form: $D_{W, Z}^{\mu v}(q)=-i\left(g^{\mu \nu}-\right.$ $\left.q^{\mu} q^{\nu} / q^{2}\right) /\left(q^{2}-m_{W, Z}^{2}\right)$, i.e., in parallel with the massless photon propagator in Landau (transverse) gauge. The photon $A$ and the $Z$-boson corrections to the Dirac fermion self-energies $\delta_{A, Z}^{i} \Sigma_{f}\left(p^{2}\right)$, $i=v, l, u, d$ are characterized by the strengths $-e^{2} Q_{i}^{2}$ and $-e^{2} P_{i Z}^{2}$, respectively, where

$$
Q_{v}^{2}=0, \quad Q_{l}^{2}=1, \quad Q_{u}^{2}=\frac{4}{9}, \quad Q_{d}^{2}=\frac{1}{9}, \quad P_{i Z}^{2}=\left(1-4 Q_{i} \sin ^{2} \theta_{W}\right)^{2} / 4 \sin ^{2} 2 \theta_{W}
$$

The $W$-boson correction to the Dirac self-energies $\delta_{W} \Sigma_{f}\left(p^{2}\right)$ is characterized by the strength which does not depend upon the charge-distinguishing index $i:-e^{2} P_{W}^{2}$ where $P_{W}^{2}=1 / 8 \sin ^{2} \theta_{W}$. For $\Sigma\left(p^{2}\right)=m^{2} / p$ the integrals of the electroweak corrections can be computed exactly.

In order to obtain a comprehensible, illustrative fermion mass formula we make approximations. The result is the Euclidean $\Sigma_{f}^{i}\left(p^{2}\right)$ :

$$
\Sigma_{f}^{i}\left(p^{2}\right)=\frac{m_{f}^{2}}{p}+\delta_{A}^{i(I R)} \Sigma_{f}\left(p^{2}\right)+\delta_{Z}^{i(I R)} \Sigma_{f}\left(p^{2}\right)+\delta_{W}^{(I R)} \Sigma_{f}\left(p^{2}\right) .
$$

The fermion masses $m_{f}^{i}$ are given as the poles of the full fermion propagators with $\Sigma_{f}^{i}\left(p^{2}\right)$ in the Minkowski space. For this reason we replace in (9) the Euclidean $p=\sqrt{ } p^{2}$ by $p=\sqrt{ }-p^{2}$. The resulting pole equation to be solved for $p^{2}=m_{f}^{i 2}$ with fixed $i, f$ has the form

$$
p^{2}=\Sigma_{f}^{i \dagger}\left(p^{2}\right) \Sigma_{f}^{i}\left(p^{2}\right) \quad \text { with } \quad \Sigma_{f}^{i}\left(p^{2}\right)=-i \frac{m_{f}^{2}}{\sqrt{ } p^{2}} A_{f}^{i}\left(p^{2}\right)+i \sqrt{ } p^{2} B_{f}^{i}\left(p^{2}\right)
$$

The real dimensionless functions $A, B$ are

$$
\begin{aligned}
A_{f}^{i}\left(p^{2}\right)=1 & +\frac{3 \alpha}{8 \pi} Q_{i}^{2}\left[1-\frac{m_{f}^{2}}{p^{2}} \operatorname{arctg} \frac{p^{2}}{m_{f}^{2}}\right] \\
& -\frac{3 \alpha}{8 \pi} P_{i Z}^{2}\left\{\frac{1}{2}\left(1-\frac{m_{f}^{2}}{m_{Z}^{2}}\right) \operatorname{arctg} \frac{p^{2}}{m_{f}^{2}}+\frac{m_{f}^{2}}{p^{2}}\left[\operatorname{arctg} \frac{p^{2}}{m_{f}^{2}}+\frac{3 m_{Z}^{4}+m_{f}^{4}}{6 m_{f}^{2} m_{Z}^{2}} \ln \left(1+\frac{p^{4}}{m_{f}^{4}}\right)\right]\right\} \\
& -\frac{3 \alpha}{8 \pi} P_{W}^{2}\left\{\frac{1}{2}\left(1-\frac{m_{f}^{2}}{m_{W}^{2}}\right) \operatorname{arctg} \frac{p^{2}}{m_{f}^{2}}+\frac{m_{f}^{2}}{p^{2}}\left[\operatorname{arctg} \frac{p^{2}}{m_{f}^{2}}+\frac{3 m_{W}^{4}+m_{f}^{4}}{6 m_{f}^{2} m_{W}^{2}} \ln \left(1+\frac{p^{4}}{m_{f}^{4}}\right)\right]\right\}, \\
B_{f}^{i}\left(p^{2}\right)= & \frac{3 \alpha}{32 \pi}\left(P_{i Z}^{2} \frac{m_{f}^{2}}{m_{Z}^{2}}+P_{W}^{2} \frac{m_{f}^{2}}{m_{W}^{2}}\right)\left[\frac{5}{3}-\ln \left(1+\frac{p^{4}}{m_{f}^{4}}\right)\right] .
\end{aligned}
$$

For an illustration, we present the approximate explicit solution of the pole equation (10) assuming $m_{f}^{2} / m_{f}^{i 2} \ll 1$, upon which the dependence on $\alpha$ disappears, ending up with

$$
m_{f}^{i}=m_{f} \exp \left\{\frac{50 \pi \sum m_{f}^{2}}{9 m_{f}^{2}\left[\left(T_{3 L}^{i}-2 Q_{i} \sin ^{2} \theta_{W}\right)^{2}+\frac{1}{2}\right]}\right\}
$$

Although aesthetically appealing this mass formula does not approximate the real world.

Also in general, with the Euclidean $\Sigma_{f}\left(p^{2}\right)=m_{f}^{2} / p$ resulting from a crude separable approximation the hopes remain unfulfilled: The numerical solutions of the equation (10) exhibit only the small, nonrealistic fermion mass splitting. 


\section{Conclusions}

We have presented a model based on the replacement of the weakly coupled Higgs sector of the SM with many parameters by the strong-coupling QFD with a single parameter. It represents so far merely a possible framework, or scenario. The most difficult step is to provide the convincing argument for $M_{f R} \gg m_{f}$. The same property was obtained by Yanagida [5] easily in a very useful model with fermion content identical to ours, but with the rich Higgs sector of elementary Higgs fields. Our fermion-composite Higgs-type operators have the same quantum numbers.

Further, it remains to compute the quantum electroweak corrections to all quantities in which the Dirac fermion masses $m_{f}$, generated by QFD, enter degenerate. Reliable computation of masses of $h, h_{3}$ and $h_{8}$ as well as the computation of other properties of the expected strongly bound QFD bound states (e.g. the dark matter right-handed neutrino composites) which are not of the NG nature requires the generically strong-coupling tools; a formidable task.

At this stage we can still make several conclusions. First of all, there is no fundamental electroweak mass scale. The only genuine scale in the game is the QFD scale $\Lambda$, phenomenologically pushed to huge values, e.g., by the seesaw mechanism for light neutrino masses or by the lack of the FCNC processes. The super-heavy Majorana neutrinos are the necessary ingredient in leptogenesis. Furthermore, the heavy composite higgses can possibly be identified with inflatons.

Our scenario predicts existence of three composite electroweakly interacting Higgs bosons $h$, $h_{3}$ and $h_{8}$ at around the scale of SM fermion masses. We believe our $h$ is the CERN SM-like Higgs boson. Derivation of its fermion-loop-generated couplings with the electroweak gauge bosons $A, W, Z$ is in progress. The model stands and falls with the existence of the Higgs-like particles $h_{3}$ and $h_{8}$, which are distinguished by the effective flavor-sensitive Yukawa couplings. This means that not all three families are quite alike.

\section{References}

[1] S. Weinberg, in CERN Courier 57, November 2017, p.31.

[2] P. Beneš, J. Hošek, A. Smetana, submitted to JHEP, arXiv:2011.03312

[3] H. Pagels and S. Stokar, Phys. Rev. D11, 2947 (1979).

[4] A. Carter and H. Pagels, Phys. Rev. Lett. 47, 1845 (1979).

[5] T. Yanagida, Phys. Rev. D20, 2986 (1979).

[6] H. Pagels, Phys. Rev. D21, 2336 (1980).

[7] J. Hosek, A model that underlies the Standard model, arXiv:1606.03292.

[8] J. S. Ball and T.-W. Chiu, Phys. Rev. D22, 2542 (1980).

[9] R. Delbourgo, Nuovo Cimento 49A, 484 (1979). 